\title{
Augmented repair of radial meniscus tear with biomimetic electrospun scaffold: an in vitro mechanical analysis
}

Benjamin B. Rothrauff ${ }^{1,4}$, Piya-on Numpaisal ${ }^{1,5}$, Brian B. Lauro ${ }^{1,3}$, Peter G. Alexander ${ }^{1}$, Richard E. Debski ${ }^{2,3,4}$, Volker Musahl $1^{2,3}$ and Rocky S. Tuan 1,3,4*

\begin{abstract}
Background: Large radial tears that disrupt the circumferential fibers of the meniscus are associated with reduced meniscal function and increased risk of joint degeneration. Electrospun fibrous scaffolds can mimic the topography and mechanics of fibrocartilaginous tissues and simultaneously serve as carriers of cells and growth factors, yet their incorporation into clinically relevant suture repair techniques for radial meniscus tears is unexplored. The purposes of this study were to (1) evaluate the effect of fiber orientation on the tensile properties and suture-retention strength of multilayered electrospun scaffolds and (2) determine the mechanical effects of scaffold inclusion within a surgical repair of a simulated radial meniscal tear. The experimental hypothesis was that augmentation with a multilayered scaffold would not compromise the strength of the repair.

Methods: Three multilayered electrospun scaffolds with different fiber orientations were fabricated-aligned, random, and biomimetic. The biomimetic scaffold was comprised of four layers in the following order (deep to superficial)-aligned longitudinal, aligned transverse, aligned longitudinal, and random-respectively corresponding to circumferential, radial, circumferential, and superficial collagen fibers of the native meniscus. Material properties (i.e., ultimate stress, modulus, etc.) of the scaffolds were determined in the parallel and perpendicular directions, as was suture retention strength. Complete radial tears of lateral bovine meniscus explants were repaired with a double horizontal mattress suture technique, with or without inclusion of the biomimetic scaffold sheath. Both repair groups, as well as native controls, were cyclically loaded between 5 and $20 \mathrm{~N}$ for 500 cycles and then loaded to failure. Clamp-to-clamp distance (i.e., residual elongation) was measured following various cycles. Ultimate load, ultimate elongation, and stiffness, were also determined. Group differences were evaluated by one-way ANOVA or Student's t-test where appropriate.

(Continued on next page)
\end{abstract}

\footnotetext{
* Correspondence: rst13@pitt.edu

${ }^{1}$ Center for Cellular and Molecular Engineering, Department of Orthopaedic Surgery, University of Pittsburgh School of Medicine, 450 Technology Drive, Room 221, Pittsburgh, PA 15219, USA

${ }^{3}$ Department of Bioengineering, Swanson School of Engineering, University of Pittsburgh, Pittsburgh, PA, USA

Full list of author information is available at the end of the article
} 
(Continued from previous page)

Results: Aligned scaffolds possessed the most anisotropic mechanical properties, whereas random scaffolds showed uniform properties in the parallel and perpendicular directions. In comparison, the biomimetic scaffold possessed moduli in the parallel $(68.7 \pm 14.7 \mathrm{MPa})$ and perpendicular $(39.4 \pm 11.6 \mathrm{MPa})$ directions that respectively approximate the reported circumferential and radial tensile properties of native menisci. The ultimate suture retention load of the biomimetic scaffold in the parallel direction $(7.2 \pm 1.6 \mathrm{~N})$ was significantly higher than all other conditions $(p<0.001)$. Biomimetic scaffold augmentation did not compromise mechanical properties when compared against suture repair in terms of residual elongation after 500 cycles (scaffold: $5.05 \pm 0.89$ mm vs. repair: $4.78 \pm 1.24$ mm), ultimate failure load (137.1 $\pm 31.0 \mathrm{~N}$ vs. 124 . $4 \pm 21.4 \mathrm{~N}$ ), ultimate elongation (12.09 $\pm 5.89 \mathrm{~mm}$ vs. $10.14 \pm 4.61 \mathrm{~mm})$, and stiffness (20.8 $\pm 3.6 \mathrm{vs} .18 .4 \pm 4.7 \mathrm{~N} / \mathrm{mm})$.

Conclusions: While multilayered scaffold sheets were successfully fabricated to mimic the ultrastructure and anisotropic tensile properties of native menisci, improvements in suture retention strength or adoption of superior surgical techniques will be needed to further enhance the mechanical strength of repairs of radial meniscal tears.

Keywords: Meniscus repair, Radial tear, Scaffold

\section{Background}

Meniscus tears involving the central region remain a formidable challenge to orthopaedic surgeons, as the absence of vasculature and a complex loading environment prevent a robust healing response (Abrams et al. 2013; Arnoczky and Warren 1983; Fox et al. 2015). Compared with vertical tears, radial/flap tears, in which there can be disruption of the circumferential fibers, are associated with articular cartilage lesions of increasing severity (Henry et al. 2012). The standard treatment of partial meniscectomy often alleviates pain and mechanical symptoms in the short term but is known to accelerate joint degeneration by increasing contact stresses (Fairbank 1948; Ode et al. 2012). Recent in vitro studies have demonstrated that contact stresses do not differ from native controls until a full-thickness radial tear exceeds $90 \%$ of the meniscus width, prompting renewed efforts to preserve meniscus structure through primary suture repair (Bedi et al. 2012; Mononen et al. 2013; Muriuki et al. 2011; Ode et al. 2012).

Clinical studies have reported variable healing rates of repaired meniscal tears involving the avascular region, attributable to differences in tear morphology, tissue quality, and surgical technique (Choi et al. 2010; Henning et al. 1991; Rubman et al. 1998). However, it remains unknown whether successful healing, defined most commonly by neotissue formation as observed by arthroscopy or MRI, restores native meniscus structure and function, thereby maintaining its chondroprotective role in the articular joint. In a canine model, Newman et al. (1989) found that "healed" radial tear defects consisted of a 3-5 mm gap of fibrovascular scar that failed to restore normal tissue mechanics. As a result, numerous surgical techniques have been explored in an effort to improve repair strength and maintain apposition of the torn edges (Beamer et al. 2015; Branch et al. 2015; Herbort et al. 2010; Lee et al. 2012; Matsubara et al.
2012). Similarly, though largely unexplored in regards to meniscal tears, scaffold sheets can minimize gap formation and augment mechanical properties of surgical repairs of musculoskeletal tissues (McCarron et al. 2010, 2012). To provide mechanical support, scaffolds must possess material properties equivalent to the native tissue, while biological support through the delivery of cells or biological agents must at minimum not compromise the integrity of the surgical repair (Aurora et al. 2012).

To that end, tissue engineering strategies including the independent or combinatorial use of cells, scaffolds, and growth factors, have been increasingly investigated as a means of enhancing the healing response (Moran et al. 2015; Yu et al. 2015). In particular, electrospun nanofibers composed of biodegradable polymers can mimic the topographical and mechanical cues of dense fibrocartilaginous tissues, driving fibrochondrogenic differentiation of seeded mesenchymal stem cells (MSCs) (Baker et al. 2010) and enhancing neotissue formation when placed within a vertical tear in vivo (Baker et al. 2012; Qu et al. 2015). Fisher et al. (2015) recently fabricated a multilayered cell-seeded nanofibrous scaffold capable of mimicking the anisotropic tensile properties of the meniscus that are derived from the complex organization of circumferential and radial tie fibers (Fox et al. 2015). Similarly, our recent report showed that a cell-seeded electrospun nanofibrous scaffold enhanced the mechanical and histological properties of an in vitro repair model of a radial meniscus tear (Shimomura et al. 2015).

Because the mechanical effect of incorporating an electrospun scaffold that structurally mimics meniscus fibrous architecture within a surgical repair is unknown, the purposes of this study were to (1) evaluate the effect of fiber orientation on the tensile properties and suture-retention strength of multilayered electrospun scaffolds and (2) determine the mechanical effects of scaffold inclusion within a 
surgical repair of a simulated radial meniscal tear. The experimental hypothesis was that augmentation with a multilayered scaffold would not compromise the strength of the repair.

\section{Methods}

Study design

Individual sheets of aligned or randomly oriented electrospun nano/microfibers were combined to form biomimetic multilayered scaffolds. In addition to fiber diameter characterization, the tensile and sutureretention properties of the scaffolds were determined. The biomimetic scaffold, modeling the fibrous structure of the native meniscus, was incorporated as a sheath enveloping the tibia and femoral surfaces of a radially transected lateral bovine meniscus. The mechanical properties of the suture repair, with or without inclusion of the biomimetic scaffold, were determined following cyclic loading and subsequent load to failure.

\section{Fabrication of multilayered nanofibrous scaffold}

Multilayered nanofibrous scaffolds were fabricated through electrospinning, as shown in Fig. 1. The electrospinning apparatus is shown in Fig. 1A-C. Three orientations of fibers-aligned longitudinal (Fig. 1D), aligned transverse (Fig. 1E), and random (Fig. 1F)-were utilized to create three designs of multilayered scaffolds-(1) aligned (Fig. 1G), (2) random (Fig. 1H), or (3) biomimetic (Fig. 1I). The biomimetic scaffold was comprised of four layers in the following order (deep to superficial): aligned longitudinal, aligned transverse, aligned longitudinal, and random. This design was inspired by the fibrous structure of the native meniscus, in which the circumferential collagen fibers resist hoop stresses (aligned longitudinal) while the tie fibers resist radial stresses (aligned transverse) and random fibers constitute the meniscal surfaces (random) (Fox et al. 2015; Makris et al. 2011). Each layer was fabricated from a solution of poly- $\varepsilon$-caprolactone $(\mathrm{PCL}, \mathrm{MW}=$ 70 k-90 kd, Sigma-Aldrich, St. Louis, MO) prepared at $15 \% \mathrm{w} / \mathrm{v}$ in $1: 1(\mathrm{v} / \mathrm{v})$ tetrahydrofuran (THF, SigmaAldrich):dimethylformamide (DMF, Sigma-Aldrich). The PCL solution was loaded into a $10 \mathrm{ml}$ syringe and extruded through an 18-gauge blunt tip needle at $3.0 \mathrm{~mL} / \mathrm{h}$ using a syringe pump (PY2 70-2209; Harvard Apparatus, Holliston, MA). The needle tip was placed $10 \mathrm{~cm}$ from a custom-designed cylindrical mandrel, which rotated at a surface velocity of $10 \mathrm{~m} / \mathrm{s}$ for aligned fibers or
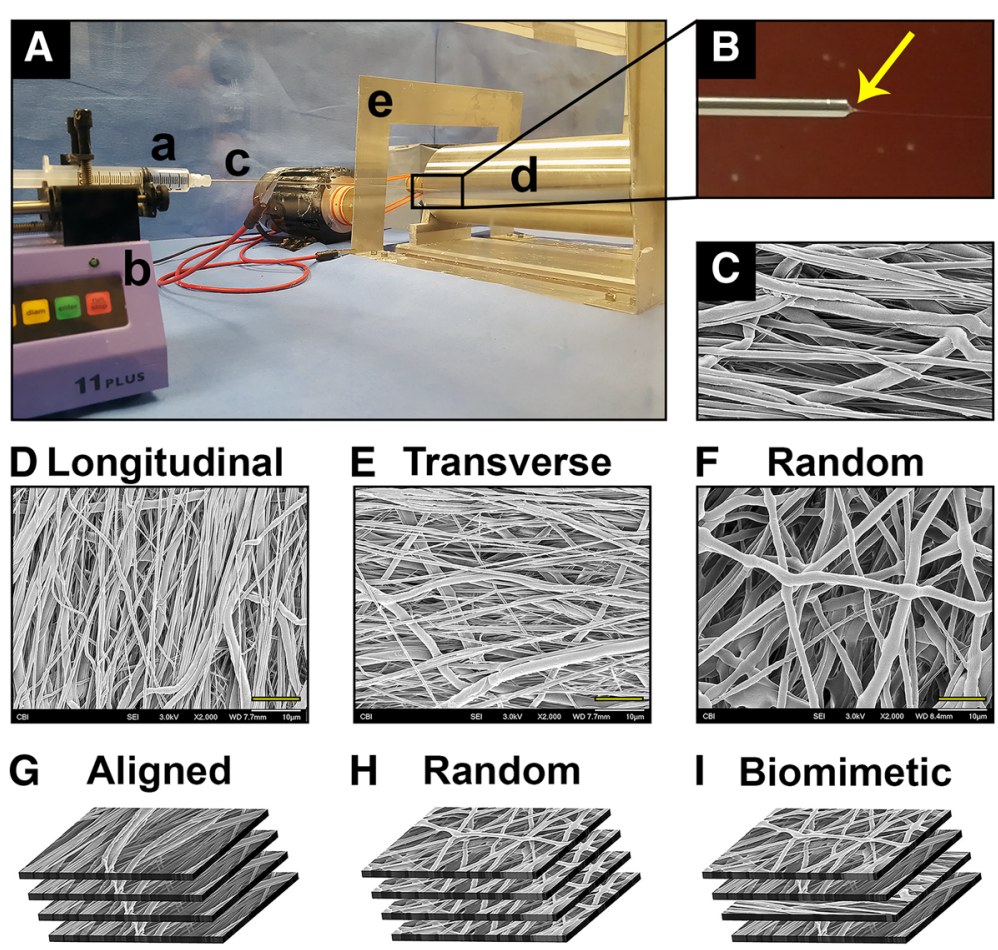

Fig. 1 Fabrication of multilayered electrospun scaffolds. (A) Electrospinning apparatus consisting of (a) syringe with polymer solution, (b) syringe pump, (c) 18-gauge blunt tip needle, (d) rotating mandrel, and (e) aluminum shield. (B) Taylor cone (arrow) with emerging polymer fiber creates (C) nanofibrous sheet. (D-F) SEM images of fiber orientations comprising individual layers. Scale bar, $10 \mu \mathrm{m}$. (G-I) Individual layers are combined to form three types of multilayered scaffolds, $(\mathbf{G})$ aligned, (H) random, and (I) biomimetic (consisting of alternating layers of aligned and random layers) 
$0.5 \mathrm{~m} / \mathrm{s}$ for random fibers. $10-18 \mathrm{kV}$ DC potential (Gamma High Voltage, Ormand Beach, FL) was applied to the polymer solution while an $8 \mathrm{kV}$ potential was applied to two aluminum shields placed perpendicular to the mandrel axis but parallel to the needle axis (Fig. 1A). For the biomimetic scaffolds, a given layer was removed, reoriented, and reattached to the mandrel such that the fibers of the subsequent layer were electrospun directly onto the former.

\section{Characterization of multilayered nanofibrous scaffold}

Average fiber diameter in each layer was determined by scanning electron microscopy (SEM). Briefly, scaffold layers were dried under vacuum and mounted on aluminum stubs, sputter-coated with $4.5 \mathrm{~nm}$ of gold, and imaged by SEM (field emission, JEOL JSM6335F, Peabody, MA) operated at $3 \mathrm{kV}$ accelerating voltage and $8 \mathrm{~mm}$ working distance. Images were morphometrically analyzed using Image J (National Institutes of Health, Bethesda, MD).

Each multilayered scaffold was cut into dumbbellshaped constructs with a central rectangular area measuring $25 \mathrm{~mm}$ by $5 \mathrm{~mm}$. Construct thickness was measured with digital calipers at three sites and averaged, from which the cross-sectional area (CSA) was calculated. Constructs were then clamped into a materials testing machine (Model 4502; Instron, Norwood, MA) and loaded under tension in a direction either parallel or perpendicular to fiber alignment. This distinction was arbitrary for random scaffolds. For the biomimetic scaffold, the scaffold was oriented with the two aligned longitudinal layers defining parallel. After preloading to $0.5 \mathrm{~N}$, constructs were preconditioned from 0 to $2 \%$ strain (estimated from clamp-to-clamp distance) for 15 cycles at $20 \mathrm{~mm} / \mathrm{min}$ before undergoing load to failure at the same elongation rate. A custom digital motion tracking system (Spica Technology, Kihei, Maui, HI; $0.01 \mathrm{~mm}$ accuracy) was used to track the vertical displacement of the strain markers (black pen) using a single video camera aligned perpendicular to the plane. These data were inputted into ABAQUS software (ABAQUS/CAE Student Version 6.4; Simulia, Providence, RI) to determine strain. Both structural and material properties were determined. Of note, stiffness (modulus) was determined from the slope of the linear region of the load-elongation (stress-strain) curve while yield load (stress) and yield elongation (strain) were found at the intersection of the data curve and the tangent line with a $0.2 \%$ positive offset along the $\mathrm{x}$-axis, as described previously (Czaplewski et al. 2014).

To determine suture retention strength, scaffolds were clamped on both ends, as in the tensile testing protocol described above, and evenly transected. A single loop of 3-0 prolene suture was passed through the midline of each construct at a distance of $5 \mathrm{~mm}$ from the cut edge and secured to a immovable cylindrical rod mounted on the material testing machine. A preload of $0.5 \mathrm{~N}$ was applied before loading to failure at $20 \mathrm{~mm} / \mathrm{min}$. The maximum load was recorded as the suture retention strength.

\section{Suture repair of radial tear of lateral meniscus}

Twenty-four fresh-frozen lateral menisci of adult cows (2-3 years old, JW Treuth \& Sons Inc., Catonsville, MD) were used to simulate repair of a radial tear. Menisci were radially transected in the midbody, beginning in the central region and extending to a width of $90 \%$, corresponding to a length of $\sim 27 \mathrm{~mm}$ (out of $30 \mathrm{~mm}$ ). A single horizontal stitch of 2-0 braided polyester suture (TiCron, Covidien, Dublin, Ireland) was placed $7 \mathrm{~mm}$ from the tear edges and in the center of the tear width to reduce the transected edges (Fig. 2a). The remaining $10 \%$ width was then transected to complete the tear. In the scaffold-augmented group, an hour-glass shaped biomimetic scaffold was wrapped around the tear site so as to cover the femoral and tibial surfaces of the
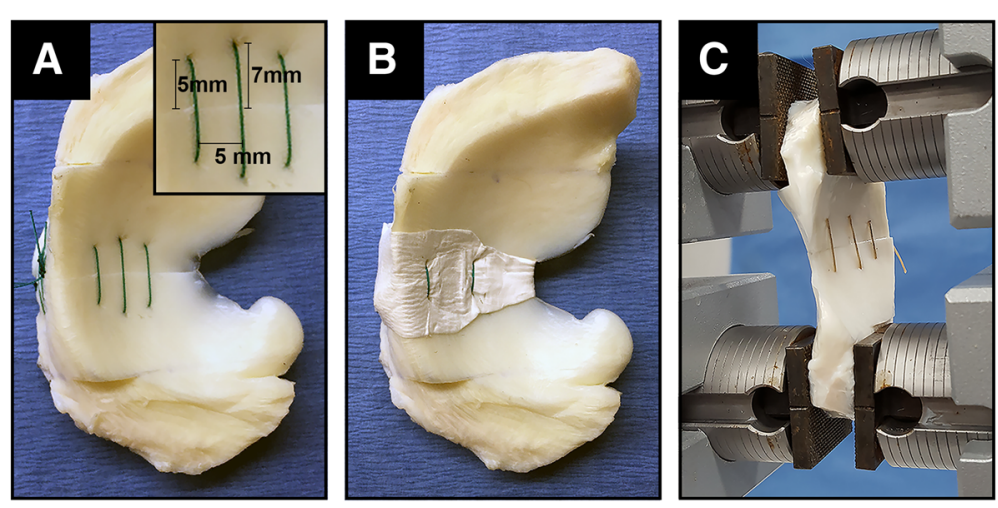

Fig. 2 Suture repair of meniscal tears and mechanical testing set-up. a Suture repair of fully transected meniscus. Inset shows dimensions of suture placement. $\mathbf{b}$ Scaffold-augmented repair. c Suture repaired meniscus clamped in materials testing machine prior to tensile loading protocol 
meniscus. The scaffold was oriented such that the two layers of aligned longitudinal nanofibers were parallel to the circumferential fibers of the meniscus. For both the suture repair and scaffold-augmented groups $(n=8$ per group), a double horizontal mattress suture technique was subsequently performed in which the sutures were positioned $5 \mathrm{~mm}$ medial and lateral to the reducing suture and $5 \mathrm{~mm}$ from the tear margin (Fig. 2a, b). The double horizontal sutures passed through, and laid superficial to, the scaffold sheath so as to ensure its stable incorporation into the repair. Native menisci served as intact controls against which both repair groups were compared.

\section{Mechanical testing of suture repair}

Three groups of menisci were tested-(1) intact controls, (2) suture repairs, and (3) scaffold-augmented repairs, as described above. For each, the anterior and posterior portions of the menisci were trimmed to provide relatively flat surfaces for clamping (Fig. 2). Menisci were clamped in a materials testing machine such that the axis of tension was perpendicular to the simulated tear (Fig. 2c). After preloading to $5 \mathrm{~N}$, the construct was cyclically loaded from 5 to $20 \mathrm{~N}$ for 500 cycles at a rate of $20 \mathrm{~mm} / \mathrm{min}$, pausing after 250 cycles to tighten the clamps. Thereafter, the construct was loaded to failure at the same rate. Residual elongation (indicative of gap formation) was determined after 1, 10, 50, 100, 250, and 500 cycles. Ultimate load, ultimate elongation, and stiffness were determined from the load to failure.

\section{Statistical analysis}

A priori power calculations utilizing pilot data and values from relevant literature revealed a minimum sample size of seven was required to detect a $15 \%$ difference in ultimate load when comparing scaffold-augmented repairs against suture repair alone (power $=0.80, \alpha=0.05$ ). As a result, eight menisci were allocated to each group for mechanical testing. In analyzing scaffold properties, a two-way ANOVA with fixed factors-scaffold type (3) and direction (2)-was employed to determine main and interactive effects. Subsequent one-way ANOVA with Tukey post-hoc tests or Student's $t$-tests were performed to determine statistical differences between conditions. One-way ANOVA with post-hoc Tukey's test was performed to evaluate differences in native meniscus, suture repairs, and scaffold-augmented repairs, after undergoing both cyclic loading and load to failure. $p<0.05$ was considered statistically significant.

\section{Results}

\section{Scaffold properties}

Three orientations of PCL fibers-(1) aligned longitudinal (fiber diameter: $811 \pm 388 \mathrm{~nm}$, Fig. 1D), (2) aligned transverse (diameter: $772 \pm 408 \mathrm{~nm}$, Fig. 1E), and (3) random (diameter: $1562 \pm 524 \mathrm{~nm}, \quad$ Fig. 1F)-were successfully prepared by electrospinning. From these orientations, three multilayered scaffolds were fabricated-(1) aligned (thickness: $0.60 \pm 0.04 \mathrm{~mm}$, Fig. 1G), (2) random (thickness: $0.37 \pm 0.08 \mathrm{~mm}$, Fig. $1 \mathrm{H}$ ), and (3) biomimetic (thickness: $0.56 \pm 0.05 \mathrm{~mm}$, Fig. 1I). Both structural and material tensile properties were determined, with the latter presented in Table 1. For all parameters tested, the aligned scaffolds possessed the greatest degree of anisotropy, i.e., a difference when comparing values in the parallel and perpendicular direction $(p<0.001)$. Conversely, random scaffolds demonstrated isotropic properties, with no significant differences between the two directions. The biomimetic scaffold, much like the native meniscus, exhibited higher values for ultimate stress $(p<0.001)$, yield stress $(p=0.002)$, and modulus $(p<0.001$, Fig. 3a), in the parallel (i.e., circumferential) direction, as compared to the perpendicular (i.e., radial). The biomimetic scaffold possessed lower material properties than the aligned scaffolds in the parallel direction, although these differences did not consistently reach statistical significance. Conversely, the biomimetic scaffold was superior to both the aligned and random scaffolds when considering the perpendicular direction, as found for ultimate stress $(p<0.001$ vs. aligned, $p=0.042$ vs. random), yield stress $(p<0.001$ vs. aligned, $p=0.046$ vs. random), and modulus $(p<0.001$

Table 1 Material Properties of Scaffold Designs ${ }^{a}$

\begin{tabular}{|c|c|c|c|}
\hline & Aligned & Random & Biomimetic \\
\hline \multicolumn{4}{|c|}{ Ultimate Stress (MPa) } \\
\hline Parallel & $12.9 \pm 4.3^{* * * *}$ & $3.4 \pm 1.1^{* * *}$ & $8.5 \pm 1.9^{*}, * * *$ \\
\hline Perpendicular & $1.2 \pm 0.3^{* * * *}$ & $3.8 \pm 1.0^{* * *}$ & $5.1 \pm 1.0^{* * *}$ \\
\hline \multicolumn{4}{|c|}{ Ultimate Strain ( $\mathrm{mm} / \mathrm{mm})$} \\
\hline Parallel & $0.40 \pm 0.03^{*}$ & $3.28 \pm 1.49^{* * * *}$ & $0.34 \pm 0.08$ \\
\hline Perpendicular & $3.69 \pm 1.30$ & $3.00 \pm 1.46$ & $0.40 \pm 0.07^{* * *}$ \\
\hline \multicolumn{4}{|l|}{ Modulus (MPa) } \\
\hline Parallel & $93.6 \pm 33.9^{*}, * * * * * *$ & $16.9 \pm 9.7$ & $68.7 \pm 14.7^{*}, * * * * * *$ \\
\hline Perpendicular & $2.7 \pm 0.5^{* * *}$ & $18.5 \pm 5.4^{* * *}$ & $39.4 \pm 11.6^{* * * *}$ \\
\hline \multicolumn{4}{|l|}{ Yield Stress (MPa) } \\
\hline Parallel & $4.9 \pm 1.5^{*, * * * * *}$ & $1.7 \pm 0.5$ & $4.0 \pm 1.3^{* * * * * * * * *}$ \\
\hline Perpendicular & $0.4 \pm 0.1^{* * * *}$ & $1.7 \pm 0.4^{* * *}$ & $2.2 \pm 0.4^{* * *}$ \\
\hline \multicolumn{4}{|c|}{ Yield Strain $(\mathrm{mm} / \mathrm{mm})$} \\
\hline Parallel & $0.06 \pm 0.01^{* * * * *}$ & $0.11 \pm 0.04$ & $0.07 \pm 0.01^{* * * * *}$ \\
\hline Perpendicular & $0.12 \pm 0.07$ & $0.10 \pm 0.04$ & $0.07 \pm 0.02$ \\
\hline
\end{tabular}

${ }^{a}$ For a given scaffold type, significant difference when comparing parallel vs. perpendicular direction, ${ }^{*} p<0.001,{ }^{* *} p<0.05$; Significantly different from both scaffolds, ${ }^{* * *} p<0.05,{ }^{* * * *} p<0.001$; Significantly different from random scaffold, ${ }^{* * * * * *} p<0.01,{ }^{* * * * * *} p<0.05$ 


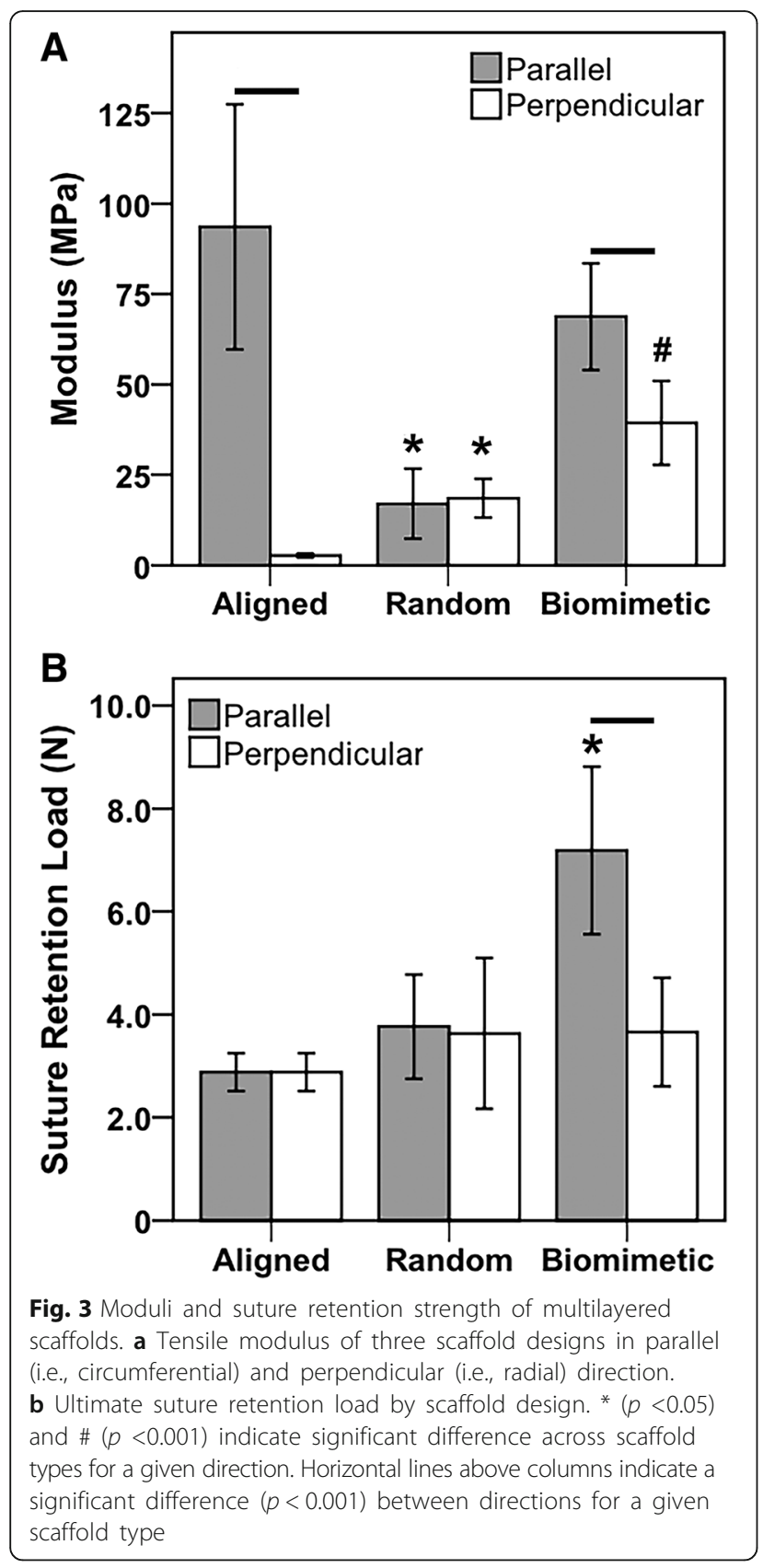

vs. both). Nevertheless, no scaffold, regardless of direction, possessed a high resistance to suture pull-through (Fig. 3b), even though the biomimetic scaffold was significantly stronger than either scaffold when oriented in the parallel direction $(p<0.001)$. This finding, coupled with its anisotropic material properties reminiscent of native menisci, supported the surgical incorporation of the biomimetic scaffold positioned such that the two layers of aligned longitudinal fibers (i.e., parallel in Table 1) were parallel to the circumferential fibers of the underlying meniscus (Fig. 2b).

\section{Meniscus repair properties}

Residual elongation values following cyclic loading are shown in Table 2. All repairs survived the cyclic loading protocol and exhibited significantly larger displacement at a given cycle when compared against native controls $(p<0.001)$. There was no statistical difference between the repair groups. Similarly, both repair groups demonstrated significantly lower ultimate failure load $(p<0.001)$ and stiffness $(p<0.001)$ than the native control, yet no differences between repair groups were found (Table 3). All repaired constructs failed by suture breakage, whereas the native meniscus specimens experienced clamp slippage.

\section{Discussion}

In this study, an electrospun scaffold that mimicked the fibrous architecture and anisotropic mechanical properties of the meniscus was fabricated. The biomimetic scaffold was incorporated within a double horizontal mattress suture repair of a complete radial tear without diminishing the mechanical properties of the repair. The biomimetic scaffold contained two layers of aligned nanofibers, imparting the greatest tensile strength in a direction parallel to these fibers, while a layer of aligned fibers oriented transversely resisted tension applied in the perpendicular direction. When used as an augmentation to meniscal repair, these fibers are intended to mimic the circumferential and radial tie fibers of the native meniscus, respectively (Fithian et al. 1990). Similarly, the layer of randomly oriented fibers mimics the surface of the native meniscus (Fithian et al. 1990; Fox et al. 2015). The anisotropic tensile properties of the biomimetic scaffold grossly matched those of native meniscus as well. Namely, the average modulus of the scaffold in the parallel (i.e. circumferential) direction was $67.8 \pm 14.7 \mathrm{MPa}$, which falls within the range of reported modulus values ( $\sim 59$ to $294 \mathrm{MPa})$ of circumferentially oriented specimens obtained from both bovine and human menisci (Fithian et al. 1990; Lechner et al. 2000; Proctor et al. 1989; Tissakht and Ahmed 1995). Similarly, the scaffold modulus in the perpendicular direction $(39.4 \pm 11.6 \mathrm{MPa})$ was within the range of native meniscus specimens ( 3 to $60 \mathrm{MPa})$ (Fithian et al. 1990; Tissakht

Table 2 Residual Elongation (mm) During 500 Cycles Between 5 and $20 \mathrm{~N}$

\begin{tabular}{llcc}
\hline Cycle & Native & Suture Repair & Scaffold-Augmented \\
\hline 1 & $0.26 \pm 0.16^{\mathrm{a}}$ & $1.14 \pm 0.28$ & $1.27 \pm 0.38$ \\
10 & $0.40 \pm 0.23^{\mathrm{a}}$ & $1.75 \pm 0.40$ & $1.99 \pm 0.33$ \\
50 & $0.55 \pm 0.33^{\mathrm{a}}$ & $2.57 \pm 0.57$ & $2.93 \pm 0.35$ \\
100 & $0.66 \pm 0.39^{\mathrm{a}}$ & $3.15 \pm 0.75$ & $3.58 \pm 0.47$ \\
250 & $0.86 \pm 0.51^{\mathrm{a}}$ & $4.29 \pm 1.17$ & $4.88 \pm 0.80$ \\
500 & $0.93 \pm 0.49^{\mathrm{a}}$ & $4.78 \pm 1.24$ & $5.05 \pm 0.89$ \\
\hline
\end{tabular}

${ }^{a}$ Native control significantly less $(p<0.001)$ than either repair group at given cycle 
Table 3 Mechanical Properties of Native and Repaired Menisci Pulled to Failure

\begin{tabular}{lccc}
\hline & Native & Suture Repair & Scaffold-Augmented \\
\hline Ultimate Load $(\mathrm{N})^{\mathrm{a}}$ & $437.3 \pm 117.5$ & $124.4 \pm 21.4$ & $137.1 \pm 31.0$ \\
$\begin{array}{l}\text { Ultimate Elongation } \\
(\mathrm{mm})^{\mathrm{b}}\end{array}$ & $5.12 \pm 1.55$ & $10.14 \pm 4.61$ & $12.09 \pm 5.89$ \\
Stiffness $(\mathrm{N} / \mathrm{mm})$ & $141.0 \pm 42.4^{\mathrm{a}}$ & $18.4 \pm 4.7$ & $20.8 \pm 3.6$ \\
\hline
\end{tabular}

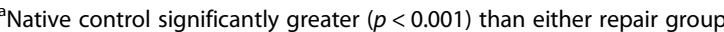

${ }^{\mathrm{b}}$ Scaffold-augmented group significantly greater $(p=0.022)$ than native control

and Ahmed 1995). As the range of values suggests, the material properties of native menisci vary broadly by region (Fithian et al. 1990; Proctor et al. 1989; Tissakht and Ahmed 1995) and are further affected by the crosssectional area of the specimen undergoing tensile testing (Lechner et al. 2000). The scaffold fabrication parameters utilized in this study could be modified to provide increased stiffness in the circumferential direction with corresponding reductions in the radial direction, as exaggerated in the aligned scaffold. The resulting moduli would then more closely match the average reported moduli of native menisci. However, the superficial regions of the menisci exhibit more isotropic properties than the deeper regions, a finding worthy of consideration when implementing a scaffold as a sheath, such as in this study (Fithian et al. 1990).

While the biomimetic scaffold mimicked the topography and tensile properties of native menisci, it did not improve the mechanical properties of a simulated radial meniscal tear repaired with suture. Both the ultimate failure load $(\sim 125 \mathrm{~N})$ and stiffness $(\sim 19 \mathrm{~N} / \mathrm{mm})$ of the repair and scaffold-augmented groups are comparable to reported values using similar suture techniques in cadaveric models (Beamer et al. 2015; Bhatia et al. 2015; Branch et al. 2015; Herbort et al. 2010). In testing different suture techniques, Herbort et al. (2010) and Branch et al. (2015) confirmed that multiple sutures are superior to a single suture loop, providing in vitro support to the clinical standard of using an inside-out double horizontal suture technique for repair of radial tears. In this study, a single horizontal stitch was placed equidistant from the central and peripheral rims to reduce the torn edges before performing a double horizontal mattress suture repair, with or without inclusion of the biomimetic scaffold serving as a sheath. As visualization of tear apposition was impossible with the opaque scaffold in place, the reducing stitch was necessary. This third stitch likely accounts for the elevated ultimate load found in this study, as compared against those reported by Herbort et al. (2010) (109 N) and Bhatia et al. (2015) $(106 \mathrm{~N})$ when a double horizontal suture technique was evaluated. Unfortunately, the scaffold also prevented direct visualization of markers that might otherwise be used to track tissue strain and gap formation, as utilized in related studies (Beamer et al. 2015; Bhatia et al. 2015). Consequently, residual elongation (i.e. clamp-to-clamp distance) was used to indicate gap formation, although the $\sim 1 \mathrm{~mm}$ elongation found after 500 cycles in the native controls suggests that clamp slippage and/or viscoelastic creep partially contributed to this measurement of gap formation.

The biomimetic scaffold of this study enveloped the tear site and was secured as part of the double horizontal suture repair. This approach, inspired by the application of scaffold sheets to augment rotator cuff repairs (Ratcliffe et al. 2015), stands in contrast to a previous clinical study in which autologous fascia was used to wrap surgical repairs of complex meniscal tears (Henning et al. 1991). Namely, the fascia sheath was not intended to provide any mechanical support to the suture repair and therefore was attached to the meniscus only along the peripheral rim. In this study, suture breakage was the mode of failure for all repairs, obviating any possible benefit of including a mechanically robust scaffold. If improvements in surgical materials or techniques were sufficient to alter the mechanism of failure to suture pull-through (as seen with rotator cuff repairs), augmentation with mechanically robust scaffolds could further enhance repair strength.

In order to provide mechanical support to the surgical repair, the scaffold should possess (1) material properties similar to that of the native tissue and (2) suture retention strength equal to, or greater than, the surgical repair (Aurora et al. 2012). While the biomimetic scaffold met the former criterion, it exhibited a poor ability to hold suture with an ultimate pullout load of $7.2 \pm 1.6 \mathrm{~N}$. Categorically, nonwoven electrospun nanofibers do not adequately hold suture. However, the implementation of textile patterns such as braiding or weaving could provide added resistance to suture pull-through (Hakimi et al. 2015; McCarron et al. 2010). To that end, future studies will explore fabrication methods capable of enhancing the suture retention strength of the biomimetic scaffold. Similarly, in vitro culture of cells on the biomimetic scaffold may increase mechanical properties through deposition of extracellular matrix proteins (Fisher et al. 2015) while also contributing to neotissue formation if localized to the lesion (Shimomura et al. 2015).

Baek et al. (2015) and Fisher et al. (2015) independently developed cell-seeded multilayered electrospun scaffolds that mimicked the anisotropic fibrous ultrastructure of the meniscus. When implanted within a vertical longitudinal tear created within an explant (Baek et al. 2016) and animal (Qu et al. 2015) model, these electrospun scaffolds promoted neotissue formation within the tear site. In contrast, the biomimetic scaffold of the present study was applied as a sheath 
enveloping the tear site. Application of the scaffold as a sheath is not biomimetic in the same sense as the former electrospun scaffolds placed within the body of the meniscus, whereby through the process of contact guidance the scaffolds may direct cells to deposit collagen fibers in the same orientation as the circumferential and radial fibers of the surrounding native tissue (Baek et al. 2016; Baker et al. 2010). On the other hand, radial tears are less amenable to the stable localization of scaffolds within the tear site as compared to longitudinal or horizontal tears, given the greater propensity for gapping when the meniscus is loaded. Therefore, the application of the scaffold as a sheath, if drawing lessons from augmented rotator cuff repairs (Aurora et al. 2012), could theoretically off-load the surgical repair while also protecting a cell-seeded construct placed within the defect site. Furthermore, we recently demonstrated in an explant model of a radial meniscus tear that a cell-seeded scaffold sheath could augment neotissue formation and associated mechanical properties through cell migration into the lesion and/or paracrine-mediated effects on the fibrochondrocytes in the native tissue (Shimomura et al. 2015). Nevertheless, the ex vivo culture conditions of our recent study did not replicate the mechanical demands or inflammatory mediators expected within an injured joint environment, tempering the generalization of these promising in vitro results to a clinical scenario. Stable integration of a scaffold sheath, mediated in part through enhanced suture retention strength, will be needed for translational success. Similarly, broad clinical adoption would likely require timely and secure fixation through an arthroscopic surgical approach. Related clinical studies have reported secure fixation of fascia sheaths (Henning et al. 1991) and collagen matrix membranes (Piontek et al. 2016) enveloping meniscus repairs, suggesting the feasibility of applying the scaffold sheet through an arthroscopic approach.

Beyond these opportunities to improve the scaffold design and implementation, there were several limitations inherent in this study. While the scaffold was fabricated to mimic the tensile properties of native menisci, its compressive and shear properties were not evaluated. In particular, a high coefficient of friction of the scaffold sheet could potentially abrade the articulating hyaline cartilage or enveloped meniscus. However, discrepancies between the friction coefficients of meniscus devices and native articular cartilage do not inevitably lead to joint degeneration, depending upon the ability of the device to support neotissue formation, including surface lubrication (Bonnevie et al. 2014; Lee et al. 2014). The immune response to the device is of similar concern. As PCL is a biodegradable, biocompatible biomaterial with a record of clinical safety (Sell et al. 2010), the scaffold sheet should not promote an adverse inflammatory response when implanted in vivo. Nevertheless, secure fixation of the scaffold to the meniscal lesion would be required to prevent dislodgement, with possible disruption of normal joint articulation. At present, these concerns can only be sufficiently evaluated in a large animal model.

An additional limitation to this study was that the mechanical properties of the surgical repair were only evaluated under tension. Although commonly employed in similar studies to evaluate suture techniques (Bhatia et al. 2015; Branch et al. 2015; Herbort et al. 2010), this protocol does not replicate how the meniscus functions in vivo. Instead, the native meniscus encounters a complex loading environment consisting of tensile, compressive, and shear forces, exerted dynamically in the context of other joint tissues such as cartilage, ligaments, and synovium. Analysis of surface contact stresses, as well as joint kinematics via robotic systems, would provide further insight into how novel repair techniques and biomaterials affect time-zero mechanics (Maher et al. 2011). Similarly, dynamic loading protocols simulating gait could provide information on the stability of biomaterials under more physiological conditions. To that end, such investigations should ideally be performed with human cadaveric samples, although homologous structure-function relationships of the meniscus exist across species (Proffen et al. 2012). Ultimately, long-term preclinical and clinical studies will be required to determine the potential benefit of promising results in vitro.

\section{Conclusions}

This study showed that a novel biomimetic scaffold fabricated by electrospinning could be incorporated into the repair of a radial meniscus tear without compromising the tensile properties of the repair. Future research will explore methods to enhance suture retention strength. Additionally, the effect of seeding the scaffolds with adult stem cells to further improve long-term durability and integration will be examined. With further modification, the scaffold presented in this study may provide a potential approach to enhance healing of meniscus tears in patients.

\section{Abbreviations \\ CSA: Cross-sectional area; DMF: Dimethylformamide; MSC: Mesenchymal stem cell; PCL: Poly- $\varepsilon$-caprolactone; SEM: Scanning electron microscopy; THF: Tetrahydrofuran}

\section{Acknowledgments}

The authors thank Matthew Miller and Gerald Ferrer for their technical assistance in mechanical testing and Dr. Bryson Lesniak for his recommendations on surgical technique.

\section{Funding}

This work was supported by the U.S. Department of Defense (W81XWH15-1-0104). Benjamin B. Rothrauff is a pre-doctoral trainee supported by an NIH Training Grant (5 T32 EB001026). 


\section{Authors' contributions}

All authors have read and approved the final submitted manuscript. Contributions are as follows: Research design, or the acquisition, analysis or interpretation of data: BBR, PN, BBL, PGA, RED, VM, RST. Drafting the paper or revising it critically: BBR, VM, RST. Approval of submitted and final versions: $B B R, P N, B B L, P G A, R E D, V M, R S T$.

\section{Competing interests}

The authors declare that they have no competing interests.

\section{Consent for publication}

Not applicable.

\section{Ethics approval and consent to participate}

Not applicable.

\section{Author details}

'Center for Cellular and Molecular Engineering, Department of Orthopaedic Surgery, University of Pittsburgh School of Medicine, 450 Technology Drive, Room 221, Pittsburgh, PA 15219, USA. ${ }^{2}$ Orthopaedic Robotics Laboratory, Department of Orthopaedic Surgery, University of Pittsburgh, 300 Technology Drive, Pittsburgh, PA, USA. ${ }^{3}$ Department of Bioengineering, Swanson School of Engineering, University of Pittsburgh, Pittsburgh, PA, USA. ${ }^{4} \mathrm{McG}$ owan Institute for Regenerative Medicine, University of Pittsburgh, Pittsburgh, PA 15219, USA. ${ }^{5}$ College of Medicine, National Taiwan University, Taipei, Taiwan

\section{Received: 6 July 2016 Accepted: 2 September 2016}

\section{Published online: 13 September 2016}

\section{References}

Abrams GD, Frank RM, Gupta AK, Harris JD, McCormick FM, Cole BJ (2013) Trends in Meniscus Repair and Meniscectomy in the United States, 2005-2011. Am J Sports Med 41:2333-2339

Arnoczky SP, Warren RF (1983) The microvasculature of the meniscus and its response to injury-an experimental study in the dog. Am J Sports Med 11:131-141

Aurora A, McCarron JA, van den Bogert AJ, Gatica JE, lannotti JP, Derwin KA (2012) The biomechanical role of scaffolds in augmented rotator cuff tendon repairs. J Shoulder Elb Surg 21:1064-1071

Baek J, Chen X, Sovani S, Jin S, Grogan SP, D'Lima DD (2015) Meniscus Tissue Engineering Using a Novel Combination of Electrospun Scaffolds and Human Meniscus Cells Embedded Within an Extracellular Matrix Hydrogel. J Orthop Res 33:572-583

Baek J, Sovani S, Glembotski NE, Du J, Jin S, Grogan SP, D'Lima DD (2016) Repair of avascular meniscus tears with electrospun collagen scaffolds seeded with human cells. Tissue Eng A 22:436-448

Baker BM, Nathan AS, Gee AO, Mauck RL (2010) The influence of an aligned nanofibrous topography on human mesenchymal stem cell fibrochondrogenesis. Biomaterials 31:6190-6200

Baker BM, Shah RP, Silverstein AM, Esterhai JL, Burdick JA, Mauck RL (2012) Sacrificial nanofibrous composites provide instruction without impediment and enable functional tissue formation. Proc Natl Acad Sci 109:14176-14181

Beamer BS, Masoudi A, Walley KC, Harlow ER, Manoukian OS, Hertz B, Haeussler C, Olson JJ, Deangelis JP, Nazarian A, Ramappa AJ (2015) Analysis of a New All-Inside Versus Inside-Out Technique for Repairing Radial Meniscal Tears. Arthroscopy 31:293-298

Bedi A, Kelly N, Baad M, Fox AJS, Ma Y, Warren RF, Maher SA (2012) Dynamic Contact Mechanics of Radial Tears of the Lateral Meniscus: Implications for Treatment. Arthroscopy 28:372-381

Bhatia S, Civitarese DM, Turnbull TL, LaPrade CM, Nitri M, Wijdicks CA, LaPrade RF (2015) A Novel Repair Method for Radial Tears of the Medial Meniscus: Biomechanical Comparison of Transtibial 2-Tunnel and Double Horizontal Mattress Suture Techniques Under Cyclic Loading. Am J Sports Med 44:639-645

Bonnevie ED, Puetzer JL, Bonassar $\amalg$ (2014) Enhanced boundary lubrication properties of engineered menisci by lubricin localization with insulin-like growth factor I treatment. J Biomech 47:2183-2188

Branch EA, Milchteim C, Aspey BS, Liu W, Saliman JD, Anz AW (2015) Biomechanical Comparison of Arthroscopic Repair Constructs for Radial Tears of the Meniscus. Am J Sports Med 43:2270-2276
Choi N-H, Kim T-H, Son K-M, Victoroff BN (2010) Meniscal Repair for Radial Tears of the Midbody of the Lateral Meniscus. Am J Sports Med 38:2472-2476

Czaplewski SK, Tsai T-L, Duenwald-Kuehl SE, Vanderby R Jr, Li W-J (2014) Tenogenic differentiation of human induced pluripotent stem cell-derived mesenchymal stem cells dictated by properties of braided submicron fibrous scaffolds. Biomaterials 35:6907-6917

Fairbank TJ (1948) Knee joint changes after meniscectomy. J Bone Jt Surg (Br) 30:664-670

Fisher MB, Henning EA, Soegaard N, Bostrom M, Esterhai JL, Mauck RL (2015) Engineering meniscus structure and function via multi-layered mesenchymal stem cell-seeded nanofibrous scaffolds. J Biomech 48:1412-1419

Fithian DC, Kelly MA, Mow VC (1990) Material properties and structure-function relationships in the menisci. Clin Orthop Relat Res 252:19-31

Fox AJS, Wanivenhaus F, Burge AJ, Warren RF, Rodeo SA (2015) The Human Meniscus: A Review of Anatomy, Function, Injury, and Advances in Treatment. Clin Anat 28:269-287

Hakimi O, Mouthuy PA, Zargar N, Lostis E, Morrey M, Carr A (2015) A layered electrospun and woven surgical scaffold to enhance endogenous tendon repair. Acta Biomater 26:124-135

Henning CE, Yearout KM, Vequist SW, Stallbaumer RJ, Decker KA (1991) Use of the fascia sheath coverage and exogenous fibrin clot in the treatment of complex meniscal tears. Am J Sports Med 19:626-631

Henry S, Mascarenhas R, Kowalchuk D, Forsythe B, Irrgang JJ, Harner CD (2012) Medial Meniscus Tear Morphology and Chondral Degeneration of the Knee: Is There a Relationship? Arthroscopy 28:1124-1134

Herbort M, Siam S, Lenschow S, Petersen W, Zantop T (2010) Strategies for Repair of Radial Tears Close to the Meniscal Rim-Biomechanical Analysis With a Cyclic Loading Protocol. Am J Sports Med 38:2281-2287

Lechner K, Hull ML, Howell SM (2000) Is the circumferential tensile modulus within a human medial meniscus affected by the test sample location and cross-sectional area? J Orthop Res 18:945-951

Lee CH, Rodeo SA, Fortier LA, Lu C, Erisken C, Mao JJ (2014) Protein-releasing polymeric scaffolds induce fibrochondrocytic differentiation of endogenous cells for knee meniscus regeneration in sheep. Sci Transl Med 6:266ra171

Lee YHD, Nyland J, Burden R, Caborn DNM (2012) Cyclic Test Comparison of AllInside Device and Inside-Out Sutures for Radial Meniscus Lesion Repair: An In Vitro Porcine Model Study. Arthroscopy 28:1873-1881

Maher SA, Rodeo SA, Potter HG, Bonassar LJ Wright TM, Warren RF (2011) A pre-clinical test platform for the functional evaluation of scaffolds for musculoskeletal defects: the meniscus. HSS J 7:157-163

Makris EA, Hadidi P, Athanasiou KA (2011) The knee meniscus: Structure-function, pathophysiology, current repair techniques, and prospects for regeneration. Biomaterials 32:7411-7431

Matsubara H, Okazaki K, Izawa T, Tashiro Y, Matsuda S, Nishimura T, Nakanishi Y, Kawamura H, Iwamoto Y (2012) New Suture Method for Radial Tears of the Meniscus Biomechanical Analysis of Cross-Suture and Double Horizontal Suture Techniques Using Cyclic Load Testing. Am J Sports Med 40:414-418

McCarron JA, Milks RA, Chen X, lannotti JP, Derwin KA (2010) Improved time-zero biomechanical properties using poly-L-lactic acid graft augmentation in a cadaveric rotator cuff repair model. J Shoulder Elb Surg 19:688-696

McCarron JA, Milks RA, Mesiha M, Aurora A, Walker E, lannotti JP, Derwin KA (2012) Reinforced fascia patch limits cyclic gapping of rotator cuff repairs in a human cadaveric model. J Shoulder Elb Surg 21:1680-1686

Mononen ME, Jurvelin JS, Korhonen RK (2013) Effects of radial tears and partial meniscectomy of lateral meniscus on the knee joint mechanics during the stance phase of the gait cycleA 3D finite element study. J Orthop Res 31: 1208-1217

Moran CJ, Busilacchi A, Lee CA, Athanasiou KA, Verdonk PC (2015) Biological Augmentation and Tissue Engineering Approaches in Meniscus Surgery. Arthroscopy 31:944-955

Muriuki MG, Tuason DA, Tucker BG, Harner CD (2011) Changes in Tibiofemoral Contact Mechanics Following Radial Split and Vertical Tears of the Medial Meniscus An in Vitro Investigation of the Efficacy of Arthroscopic Repair. J Bone Jt Surg (Am) 93A:1089-1095

Newman AP, Anderson DR, Daniels AU, Dales MC (1989) Mechanics of the healed meniscus in a canine model. Am J Sports Med 17:164-175

Ode GE, Van Thiel GS, McArthur SA, Dishkin-Paset J, Leurgans SE, Shewman EF, Wang VM, Cole BJ (2012) Effects of Serial Sectioning and Repair of Radial Tears in the Lateral Meniscus. Am J Sports Med 40:1863-1870

Piontek T, Ciemniewska-Gorzela K, Naczk J, Jakob R, Szulc A, Grygorowicz M, Slomczykowski M (2016) Complex Meniscus Tears Treated with Collagen 
Matrix Wrapping and Bone Marrow Blood Injection: A 2-Year Clinical FollowUp. Cartilage 7:123-139

Proctor CS, Schmidt MB, Whipple RR, Kelly MA, Mow VC (1989) Material properties of the normal medial bovine meniscus. J Orthop Res 7:771-782

Proffen BL, McElfresh M, Fleming BC, Murray MM (2012) A comparative anatomical study of the human knee and six animal species. Knee 19:493-499

Qu F, Pintauro MP, Haughan JE, Henning EA, Esterhai JL, Schaer TP, Mauck RL, Fisher MB (2015) Repair of dense connective tissues via biomaterial-mediated matrix reprogramming of the wound interface. Biomaterials 39:85-94

Ratcliffe A, Butler DL, Dyment NA, Cagle PJ Jr, Proctor CS, Ratcliffe SS, Flatow EL (2015) Scaffolds for Tendon and Ligament Repair and Regeneration. Ann Biomed Eng 43:819-831

Rubman MH, Noyes FR, Barber-Westin SD (1998) Arthroscopic repair of meniscal tears that extend into the avascular zone-A review of 198 single and complex tears. Am J Sports Med 26:87-95

Sell SA, Wolfe PS, Garg K, McCool JM, Rodriguez IA, Bowlin GL (2010) The Use of Natural Polymers in Tissue Engineering: A Focus on Electrospun Extracellular Matrix Analogues. Polymers 2:522-553

Shimomura K, Bean AC, Lin H, Nakamura N, Tuan RS (2015) In Vitro Repair of Meniscal Radial Tear Using Aligned Electrospun Nanofibrous Scaffold. Tissue Eng A 21:2066-2075

Tissakht M, Ahmed AM (1995) Tensile stress-strain characteristics of the human meniscal material. J Biomech 28:411-422

Yu H, Adesida AB, Jomha NM (2015) Meniscus repair using mesenchymal stem cells-a comprehensive review. Stem Cell Res Ther 6:86

\section{Submit your manuscript to a SpringerOpen ${ }^{\circ}$ journal and benefit from:}

- Convenient online submission

- Rigorous peer review

- Immediate publication on acceptance

- Open access: articles freely available online

- High visibility within the field

- Retaining the copyright to your article

Submit your next manuscript at $\gg$ springeropen.com 